Relations industrielles

Industrial Relations

\title{
Industry and Humanity, par William Lyon Mackenzie King, Toronto, The University of Toronto Press, 1973, 354 pp.
}

\section{Jean-Louis Langevin}

Volume 29, numéro 1, 1974

URI : https://id.erudit.org/iderudit/028493ar

DOI : https://doi.org/10.7202/028493ar

Aller au sommaire du numéro

Éditeur(s)

Département des relations industrielles de l'Université Laval

ISSN

0034-379X (imprimé)

1703-8138 (numérique)

Découvrir la revue

Citer ce compte rendu

Langevin, J.-L. (1974). Compte rendu de [Industry and Humanity, par William Lyon Mackenzie King, Toronto, The University of Toronto Press, 1973, 354 pp.] Relations industrielles / Industrial Relations, 29(1), 230-231.

https://doi.org/10.7202/028493ar

Tous droits réservés @ C Département des relations industrielles de l'Université Laval, 1974
Ce document est protégé par la loi sur le droit d'auteur. L'utilisation des services d'Érudit (y compris la reproduction) est assujettie à sa politique d'utilisation que vous pouvez consulter en ligne.

https://apropos.erudit.org/fr/usagers/politique-dutilisation/ 
teur élimine la participation de la politique de main-d'oeuvre aux objectifs économiques. Toutefois, cette ligne de pensée recouvre assez bien l'idée prédominante des décisions en main-d'oeuvre depuis le passage de l'Economic Opportunity Act en 1964.

Dans l'esprit du lecteur, il peut y avoir confusion entre politique de maind'oeuvre et formation professionnelle, Thurrow ne faisant pas la distinction; ensuite, lorsqu'il parle du revenu «gagné » comme élément d'équité, il n'est pas certain que le revenu relatif se soit vraiment accru: des effets d'augmentation dans la concurrence pour des classes d'emplois dits «secondaires 》, des effets régressifs dans l'imposition de ces faibles revenus «non-imposables》 sous le couvert de taxes à la consommation, ne sont pas étrangers au phénomène des écarts de revenus. Enfin, après les tentatives de définition du chômage selon les causes, les effets ou encore les mesures correctives, Thurrow nous présente une distinction originale basée sur la valeur sociale accordée aux coûts de réduction du chômage : c'est sans doute la définition la plus franche qu'on en ait donnée jusqu'à présent.

C'est également, comme nous le disions plus haut, ce qui différencie son point de vue de ceux de Gordon et Holt: ceux-ci s'en tiennent à une vision générale du fonctionnement du marché du travail, ce dernier considère des choix sociaux.

Le texte de Levitan est le seul qui s'appuie sur une définition du rôle et des buts de la politique de main-d'oeuvre américaine et qui traite de ses limites pratiques dont peut-être la plus importante est l'insignifiance relative des montants qui y sont consacrés, ce qui lui fait conclure : «If manpower programs ease the unemployment-inflation tradeoff, so much the better $\gg$ (p. 116). Enfin. en plus de l'intérêt que peut présenter pour un public nord-américain l'expérience suédoise, un pays qui a délibérément opté pour la recherche du pleinemploi, on trouvera dans l'article de Meidner et Anderson des considérations qui élargissent les concepts de chômage et de participation à l'emploi.
Tout au long du livre, on y sent la relativité des explications et des positions, mais il n'en demeure pas moins que Manpower Programs in the Policy Mix est sans doute le meilleur ouvrage paru à date sur les relations entre les programmes de main-d'oeuvre et la position de la Courbe de Phillips. La portée des études des quatre auteurs américains reste toutefois fort limitée et pour le moment spéculative, tant qu'on n'aura pas approfondi davantage par la recherche empirique les relations proposées. Elle éclaire cependant d'un angle nouveau la théorie du marché du travail sous-jacente aux actions prises par nos gouvernants en main-d'oeuvre, du moins théoriquement. L'ouvrage sera un point de repère précieux pour ceux qui s'intéressent ou sont impliqués dans la politique de main-d'oeuvre et l'analyse du marché du travail.

Jacques MERCIER

Université Laval

Industry and Humanity, par William Lyon Mackenzie King, Toronto, The University of Toronto Press, 1973, $354 \mathrm{pp}$.

Pour la plupart d'entre nous, William Lyon Mackenzie King a été premier ministre du Canada de 1921 à 1926 et l'on ignore ou oublie souvent qu'il a d'abord fait carrière comme expert en relations industrielles et consultant auprès du gouvernement canadien et de plusieurs grandes corporations américaines. Et c'est à la fin de sa carrière à la Fondation Rockfeller et au moment où il allait faire ses débuts comme leader du parti libéral national qu'il écrit Industry and Humanity dont la publication originale, pour être plus précis, remonte à 1918 .

Replacer Industry and Humanity dans le contexte de l'industrie d'avant et pendant la première guerre, et l'évolution de la pensée de King ne rendrait certes pas hommage à David Jay Bercuson qui signe l'introduction de cette réédition, réalisée par les Presses de l'Université de Toronto. Dans cette présentation re- 
cherchée, Bercuson introduit à la fois «l'étude des principes sous-tendant la reconstruction du monde des relations du travail » et un Mackenzie King, réformiste, qui tente d'indiquer la voie d'un changement d'attitude dans les relations industrielles et de suggérer des moyens par lesquels un nouvel esprit pourrait pénétrer l'industrie.

En plus d'un large exposé pour l'établissement d'un nouveau programme de démocratie dans le monde industriel, l'ouvrage passe en revue plusieurs crises d'importance dans les relations patronales-ouvrières de l'Amérique du Nord et révèle l'arrière-plan de multiples pièces de la législation canadienne en matière de sécurité sociale.

Lorsqu'on fait abstraction de l'attitude paternaliste de King et des rumeurs à l'effet qu'il soit l'un des auteurs du plan Mohawk, véritables «tables de la loi » et programme détaillé de la répression à l'égard des travailleurs en grève, Mackenzie King apparaît comme un des penseurs, dignes d'intérêt, en matière de relations du travail. Qu'il nous suffise de souligner que pour lui, et c'est là un des fondements de sa thèse, le monde industriel comporte non pas deux (2) ou trois (3) mais quatre (4) parties: le travail, le capital, le management et la communauté. Croyant que la démocratie et la paix industrielles puissent reposer sur la bonne foi, le raisonnement et la compréhension mutuelle, il explique qu'une bonne part de la tension, le malaise et l'injustice sociale sont crées simplement par le fait que travail et capital ne réussissent pas ou refusent de reconnaître la légitimité des intérêts des deux autres parties. Partant de là, il énonce des principes que le temps et l'expérience sauraient mettre à l'épreuve notamment que les conflits trouveraient solution par le biais d'enquêtes conjointes (où seraient présentes les quatre (4) parties) utilisant des méthodes scientifiques tout comme les crises internationales pourraient être résolues à la Société des Nations (!!!).

Ce sont là des pensées que d'aucuns pourront qualifier de pédantes, moralistes ou même farfelues, mais qu'il vaut sûrement la peine de lire dans un ouvrage qui pour certains constitue un classique.
On doit cependant prévenir le lecteur que les longueurs, les raisonnements tortueux et le style lourd de King rendent l'ouvrage difficile à lire, mais cela ne devrait pas décourager celui qui s'intéresse à l'évolution de la pensée des systèmes industriels car qu'on le veuille ou non, William Lyon Mackenzie King a écrit quelques pages de notre histoire.

Jean-Louis LANGEVIN

Université Laval

The Winnipeg General Strike, par D.G. Masters, Toronto, University of Toronto Press, 1973, 159 pp.

La grève générale de Winnipeg a été un événement important dans l'histoire du syndicalisme au Canada. En raison de son caractère syndical et politique, elle a donné lieu à de nombreuses études et à diverses interprétations.

L'ouvrage de D.C. Masters publié en 1950 demeure le classique sur le sujet. Epuisé depuis plusieurs années, il faut se réjouir de constater que les éditeurs aient décidé d'en faire une nouvelle édition brochée à prix populaire.

On s'est contenté de reprendre exactement le même texte en ajoutant seulement une brève préface de l'auteur dans laquelle celui-ci apporte quelques précisions sur des points de détail controversés.

Gérard DION

\section{Université Laval}

Labour Economics: Wages, Employment and Trade Unionism, by Allan N. Cartter and F. Ray Marshall, Homewood, Illinois, Richard D. Irwin, Inc., (Revised edition), 1972, 594 pp.

This book is a revised edition; the first edition appeared in 1967. It is aimed at the introductory labour economics and industrial relations courses. The book is divided into three major sections: comparative labour movements (about 147 pages); wages, employment and collective bargaining 